\title{
Identifying, Visualizing and Comparing Regions in Irregularly Spaced 3D Surface Data
}

\author{
Matthew J. Thurley ${ }^{*, 1}$, Kim C. Ng \\ Monash University, Electrical $\&$ Computer Systems Engineering
}

\begin{abstract}
Image segmentations have been performed to identify the surface fragmentation of rock piles using 3D surface data, and quantified. The advantages for fragmentation measurement using image analysis are significant and include: quantifying image segmentation performance in isolation of the downstream processes of fragment classification and size distribution calculation, utilisation of 3D data to overcome various limitations of photographic-based image analysis, and the capacity to use 3D fragment data to eliminate the misclassification of partially visible fragments as smaller entirely visible fragments. The segmentation results have been quantified by comparison with the 3D surface data of each individual rock fragment. Mathematical morphology and image segmentation algorithms have been extended from greyscale image based definitions and applied to irregularly spaced 3D coordinate surface data. 3D coordinate surface data can now be morphologically processed directly in $3 \mathrm{D}$, segmented, visualized and directly compared to the actual surface fragmentation in order to quantify the results.
\end{abstract}

Key words: segmentation, mathematical morphology, 3D visualization, range data

\section{Introduction}

Rock breakage is a major component of all mining and quarrying operations. Rock size affects productivity, from the initial phase of drilling and blasting, through excavation, transportation and rock crushing.

* Corresponding author.

Email addresses: matthew_thurley@yahoo.com.au (Matthew J. Thurley), kim.c.ng@eng.monash.edu.au (Kim C. Ng).

1 The presented work is part of the postgraduate thesis of Matthew J. Thurley and has been partially funded by CSIRO Exploration \& Mining. 
The purpose of drilling and blasting is to fracture solid rock in preparation for excavation, hauling and crushing. As a rock face is blasted, it falls into a pile of rocks known as a "muckpile". The resultant muckpile density and fragment size distribution must be compatible with the excavation, transportation and crushing equipment. An overly dense muckpile and overly large rock fragments hinder the operation of primary loaders such as shovels and hydraulic excavators. This leads to a reduction in the loading rate of material and an increase in wear and damage to the machinery. In addition, oversize rocks cost additional money for secondary breakage. Larger rock fragments affect transportation productivity as they reduce the tonnage of material that trucks can carry. The crushing phase is hindered by oversize rocks as they cause damage to crushers and increase down-time, reducing productivity. Furthermore, "excessive flyrock (small rock) is a safety hazard as well as a source of damage to property and equipment", Grant et al. [1].

Kojovic et al. [2], demonstrated that "an increase in the cost of drilling and blasting is swamped by the downstream benefits of improved fragmentation, with the greatest impacts being on excavation costs and crushing costs". More specifically, the study demonstrated more than an order of magnitude saving in these downstream activities compared to the additional expenditure on drilling and blasting. This study concludes that "explosive breakage is by far the most cost-effective means of breaking rock". Furthermore, Maerz et al. [3] state that "fragmentation is so closely related to the economics of the quarrying operation, it needs to be measured quickly and accurately in order to monitor blasting, and to optimize blast design". These issues define blasting optimization as critical to overall mining costs and production.

Therefore, in order to provide feedback to, and subsequently improve the productivity of the drilling and blasting phase, we seek to quantify the rock-size distribution of the produced muckpile. Sieving (or screening) is the standard method used to size rocks in mines and according to Wang and Stephansson [4] "is accomplished by passing a known weight of sample material successively through finer sieves and weighing the amount collected on each sieve to determine the percentage weight in each size fraction." However, "sieving ... is prohibitively slow and expensive for full scale production blasts" Maerz et al. [3], and the "screening of muckpiles not feasible as a routine assessment tool", Grainger and Paine [5].

Image analysis techniques promise a quick, inexpensive and non-contact solution to determining the size distribution of a rock pile. Such techniques capture information of the surface of the rock pile which is then used to infer the pile size distribution.

We separate the problem of determining the rock pile size distribution into three subproblems as follows: perform a segmentation of the surface data to 
determine the individual fragments, classify the segmented fragments into a surface size distribution, and determine the relationship between the size distribution of the surface fragments and the pile size distribution. We present research into the first of these subproblems and report on image segmentations of 3D surface data carried out to determine the surface fragmentation of rock piles.

\section{Review of Fragmentation Measurement using Image Analysis}

Image analysis as a technique for fragmentation measurement of rock piles has been the subject of research since at least Maerz et al. [3] in 1987. In 1996 the Fragblast 5 conference held a workshop on the measurement of blast fragmentation with the stated aim "to review the state-of-the-art in image processing as applied to measurements of rock fragmentation" Franklin et al. [6]. The proceedings of this workshop provide the most thorough review of the field to date.

Various photographic and image analysis techniques have been presented and applied to the identification of visible rock fragments, including Dahlhielm [7], Downs and Kettunen [8], Havermann and Vogt [9], Kleine and Cameron [10], Maerz [11], Maerz et al. [12], Schleifer and Tessier [13, 14], Wang and Stephansson [15] and the technique published by Kemeny et al. [16] and subsequently implemented and extended by Kemeny [17], Girdner et al. [18], and Kemeny et al. [19]. These techniques are variously based on greyscale and binary image analysis techniques including edge detection, thresholding, equalization, binary image morphology, region growing, and watershed segmentation.

All of these image analysis techniques use photographic greyscale images to capture the surface of a rock pile, and determine the size distribution from this sample.

\subsection{Photographic Limitations}

Photographic techniques are dependent on ambient lighting conditions and capture images with fragment shadows. According to Maerz [20], "proper lighting is essential for automated edge detection of rock fragments" and "this includes both intensity, uniformity, and contrast". Palangio and Franklin [21] indicate that each system should "aim to provide uniform indirect or diffuse lighting and avoid excessive shadows" as "most photoanalysis systems work best when each fragment is equally bright and is surrounded by a thin, uniform shadow." Clearly shadows obscure portions of the rock pile surface and 
without ideal lighting conditions, limit the visible size of some fragments and completely hide others.

In addition, uniform rock texture on neighbouring fragments in the image can cause fragment separation problems and overlapping fragments bias the sample as it can be impossible to distinguish a small fragment from a partially visible larger fragment.

By using 3D surface data we can overcome the dual problems of shadows obscuring the surface data and uniform rock texture hiding depth variation on the surface of the pile. In addition, depth variation in the data allows us to distinguish between entirely and partially visible fragments. As a result we are not subject to the bias from these sources inherent in photographic techniques.

\subsection{Overview of Existing Techniques}

SPLIT is a commercialized imaging system and one of the most prolific in the published literature, including Kemeny et al. [16], Kemeny [17], Girdner et al. [18], and Kemeny et al. [19]. Girdner et al. [18] summarizes the SPLIT image analysis steps of greyscale equalization, sobel edge detection, conversion to binary using thresholding, distance transform on the binary edge image, and watershed segmentation modified to prevent over-segmentation.

SPLIT forms a best fit ellipse for each identified region and computes the size of each fragment using the major and minor axes of the ellipse as input to a correction function developed through experimentation. The correction function has evolved over the years and was different in the following three instances: Kemeny et al. [16, pg. 1152], Kemeny [17, pg. 1283] and Girdner et al. [18, pg. 103]. This may have been impacted by the different rock type, Kemeny [17], and changes to the segmentation algorithm, Girdner et al. [18].

The involvement of the segmentation algorithm and the correction function cannot be separated, and subsequently understood, without quantifying the results of the image analysis by comparing it back to the actual surface rock fragmentation. In this system any change in results provided by the segmentation is only identified by a change in results from the correction function. Without separating these factors the capability of the image analysis techniques, or correction function cannot be quantified.

WIPFRAG is a commercialized imaging system, well published with numerous direct and associated publications including Maerz [20], Maerz [11], Maerz et al. [12], and Maerz and Zhou [22]. Maerz et al. [12] summarizes the WIPFRAG image analysis process of block identification by thresholding, gradient edge 
detection, unspecified edge reconstruction techniques, possible manual editing, and a final stage of calculating the diameter of the circle of equal area for each identified region.

FRAGSCAN is a commercialized imaging system based on publications by Cheimanoff et al. [23] and Schleifer and Tessier [13, 14]. Schleifer and Tessier [13] summarize the FRAGSCAN image analysis process of local and global equalization, thresholding, and successive morphological openings. Morphological openings are performed with predefined circular structuring elements in an effective simulated sieving technique that classifies each region in accordance with the circular structuring element size that removes it from the image. Fragment size is extrapolated from the classified circular structuring element size to spheres of equal diameter.

The CIAS imaging system, Downs and Kettunen [8] uses edge detection, an unspecified proprietary algorithm for segmentation, followed by determination of the longest and shortest line lengths across identified fragments. However, no further explanation of the image analysis method or analysis of results is provided.

The on-line IPACS system Dahlhielm [7] uses an initial low-pass filter to remove noise, a process called curve surface thresholding that examines the contours of the rock fragments and eliminates small regions, a thresholding and enhancement step, segmentation based on a $2 \times 2$ partition mode test, followed by background splitting, region merging, and pixel aggregation. An example of the application of IPACS to fragmentation measurement is provided but the results are not analysed or quantified.

Havermann and Vogt [9] present the TUCIPS imaging system that applies edge detection, thresholding, custom contour closing and object filling. Classification is based on the conversion of identified fragment area into equivalent spherical diameter, presumably by using the intermediate of a circle of equivalent area but this is not specified.

In a similar problem Frydendal and Jones [24] presented image analysis techniques for identifying sugar beets on the surface of a pile. This is a similar problem to a rock pile, simplified due to the limited variation in size and shape of the sugar beets. Furthermore, the morphological image analysis techniques applied by Frydendal and Jones [24] are of particular interest specifically as they were applied to a 3D representation of the pile surface. They used a greyscale image where the intensity represented the depth or range coordinate of the data. Frydendal and Jones [24] applied morphological edge detection and watershed segmentation followed by a graph theory approach to determine the entirely visible sugar beets. However, the actual surface segmentation of the pile was not established and no quantified measure of the segmentation 
error was made. The approach used by Frydendal and Jones [24] presents several techniques that appear useful for the segmentation of rock fragments.

\subsection{Review Summary}

The keynote address of the Fragblast 5 workshop by Cunningham [25] is particularly rigorous in its review of the published image analysis systems for rock fragmentation and in highlighting common conclusions.

Cunningham [25] noted "that almost every such paper includes a statement such as the system is practical for suitable use ... although further work is required". Furthermore, "graphs of system output versus sieving results are described as providing good or reasonable correlation" but that "it is often difficult to concur with the sentiments expressed."

The lack of detail and rigor in quantifying the results of the image analysis processes hampers further research in this field. Particularly it is worth noting that none of the cited papers quantify the image analysis results by comparing against what was actually present on the surface of the pile when the images were taken. Comparison is typically made between the sieving results and the estimates of pile size distribution determined from the image analysis.

Comparisons have been published between photographic methods and sieving, specifically Wang and Stephansson [4] and Fernlund [26]. Wang and Stephansson [4] perform image analysis on overlapping fragments and report "a systematic error compared to sieving analysis". Such a comparison is dependent not only on the image analysis techniques applied but also on the accuracy of the resultant image segmentation.

Comparing results from photographic-based methods to sieving does not provide a quantitative comparison of the image segmentation performed as it remains undistinguished from the subsequent algorithms applied. Specifically, algorithms used to convert regions in an image into measurements of fragment size, and convert the surface fragment distribution into estimates of the pile size distribution.

Maerz and Zhou [22, pg. 418] perform a comparison against a manually edited segmentation for various automatically generated segmentations but the comparison is determined on the median weight fragment of the resultant size distribution. This does not provide a quantitative analysis of the image segmentation performed as it remains undistinguished from the algorithms used to convert regions in an image into measurements of fragment size and weight.

Without directly quantifying the image segmentation results, the reader has 
little basis upon which to evaluate and compare the presented image analysis techniques.

Liu and Tran [27] provide the only rigorous analysis of any of the published fragmentation measurement systems in their experimental comparison of SPLIT, WIPFRAG and FRAGSCAN. The results of Liu and Tran [27] allow us to evaluate these systems as a whole in the presented conditions but we are still unclear as to relative merit of the image analysis techniques or the classification techniques presented.

Perhaps the most important issue evident from this review is the need to separate the problem of fragmentation measurement into manageable and most importantly, quantifiable sub-problems.

P.1 Identifying the fragments on the surface of the pile using image analysis is a well known problem, although it has not been adequately quantified.

P.2 Determining what an identified region in the image can indicate about the actual rock fragment, is again well known but needs to be separated from the variability of preliminary image analysis steps.

P.3 The initial problem is however, to determine what the surface fragmentation indicates about the size distribution of the overall pile.

It is important to emphasize that if the surface of the pile is not in some measurable way, representative of the size distribution of the pile, then no matter how accurate the techniques we use to measure and interpret the surface, the results can have little relevance to the size distribution of the pile.

Subproblem P.1 is the subject of this paper and all three of these issues are addressed in the Thurley [28] Ph.D. thesis. Furthermore, subproblem P.3 is addressed by Thurley and $\mathrm{Ng}$ [29].

\section{3D Surface Data Collection}

Segmentation of 3D surface data has not been previously been applied to the problem of fragmentation measurement of rock piles.

Actively illuminated 3D measurement techniques have several advantages over photographic based techniques. They are not dependent on ambient lighting conditions and subsequent shadowing in the field of view. There is a direct measure of $3 \mathrm{D}$ which is not subject to surface texture variation that can bias determination of fragment edges from photographic images.

3D surface data of rock piles has been collected using the Monash Shape Measurement system developed by Alexander and $\mathrm{Ng}$ [30]. This system is 
based on active triangulation and uses a projector to impose a pattern of light stripes upon the target scene which is viewed by a CCD camera. The camera position is separated from the projector in a direction perpendicular to the line of sight of the projector, and perpendicular to the projected light stripes. Figure 1 shows a diagram of the camera and projector setup. The projector imposes 128 parallel light stripes upon the target scene which is viewed by the 512 by 512 pixel CCD camera. The projector uses binary coded patterns to resolve the correspondence problem due to occlusions.
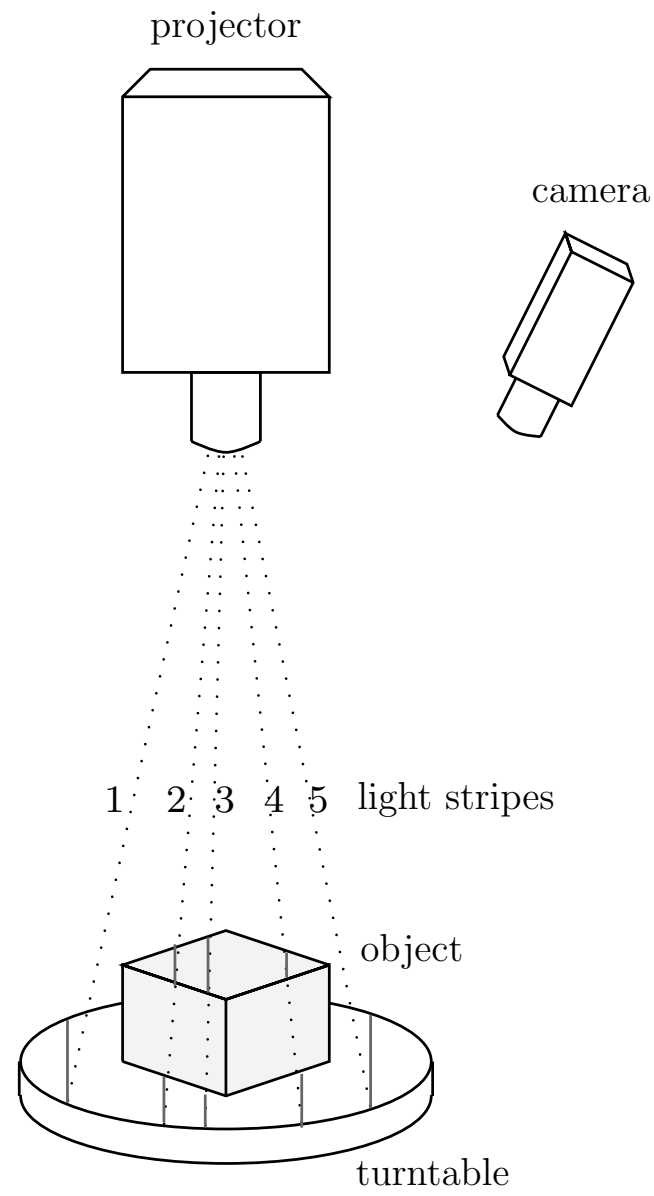

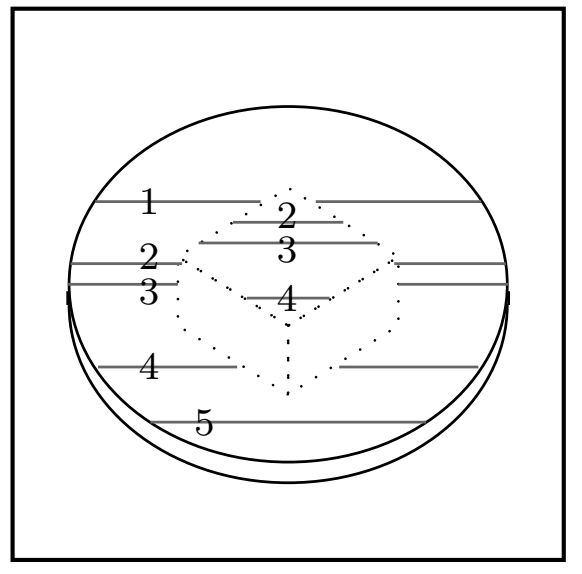

camera view

(with stripe numbers)

Note that portions of stripes 1 , 2 and 3 on the turntable are occluded in the camera view.

Fig. 1. Monash Shape Measurement System arrangement.

The orientation and location of the camera and projector are calibrated using a calibration cube and automated calibration software.

Each scan performed by the Monash Shape Measurement system, collects a 512 by 512 pixel greyscale camera image and a grid of 128 rows by 512 columns of $3 \mathrm{D}$ coordinate points. The $3 \mathrm{D}$ coordinate points are calculated from the camera image of the stripe pattern on the target scene using the known calibrated geometry between the camera and projector.

Due to the topology of the target scene and the distance between the camera 
and the projector, portions of the stripes are occluded from the camera's point of view. Figure 1 provides an example of occluded stripes with stripe 1,2 and 3 in the camera view being partially hidden behind the pictured cube. Due to the occlusion of portions of the stripes there are missing coordinate points in the collected 3D coordinate data.

The resultant 3D coordinate data from a single scan incorporates a sampling density bias of four times the density of points along the rows compared to between rows. In addition, if we consider that the target scene lies in the $\mathrm{x}-\mathrm{y}$ plane, with depth variation being represented along the $\mathrm{z}$ axis, we note that the coordinate points are irregularly spaced along the $\mathrm{x}$ and $\mathrm{y}$ axes. Each of the collected 3D coordinate points has a corresponding row and column pair, where the row indicates the stripe number and the column indicates the camera pixel number along that stripe.

We note that the Monash Shape Measurement system is not proposed as a field scanning system as structured white light is not suitable outdoors or at large scales. However, the Monash system is suitable at the laboratory scales used in this research and allowed the presented image analysis techniques to be developed within the scope of a minimal research budget. A system suitable in an industrial or mining environment would be that based on a suitable scanning laser range finder The presented image analysis techniques are readily applicable to any collection of 3D coordinate surface data.

\section{Surface Fragmentation Experiments}

The rock pile was constrained within a cylindrical bucket of diameter $265 \mathrm{~mm}$ and contained rocks of three size fractions as shown in table 1 . The rocks used in this research were blasted and crushed basalt rock fragments, sharply angular, rough in texture, and varying between block-like, plate-like, and rodlike in shape. The rocks were graded into size fractions using hand sieves containing square holes with side lengths of 11.2, 16.0, 22.4, 31.5 millimetres respectively, and then the rocks of each size class were painted a specific color to identify the corresponding sieve size of each rock.

As the data produced from a single scan of this system contains areas of occlusion, multiple scans of the rock pile have been taken from differing orientations. All scans are registered and transformed to a global 3D coordinate system. The experimental rock pile was placed upon the turntable represented in figure 1 and six scans were performed at rotations of $0,45,135,180,225$, and 315 degrees. The resultant surface model contains six overlapping sets of data totaling around 150,000 3D coordinates, as shown for the $020117 \mathrm{~b}$ data set in figure 12 (page 32). The model also contains six greyscale images, one of 
Table 1

Experimental Rock pile size classes and proportions

\begin{tabular}{|cccccc|}
\hline Sieve Size & No. & Weight & \multicolumn{2}{c|}{ Vol. } & \multicolumn{2}{c|}{ Size Class } \\
$(\mathrm{mm})$ & & (grams) & $\%$ & color & $i$ \\
\hline $11.2-16.0$ & 2000 & 8718 & 52.6 & pink & 1 \\
$16.0-22.4$ & 500 & 4709 & 28.4 & white & 2 \\
$22.4-31.5$ & 100 & 3135 & 18.9 & yellow & 3 \\
\hline
\end{tabular}

which is shown overlayed on the $3 \mathrm{D}$ data set in figure 2 with a closeup of the $3 \mathrm{D}$ coordinate points corresponding to the central large fragment in figure 3. The surface of the rock pile is oriented such that it lies in the $\mathrm{x}, \mathrm{y}$ plane with depth variation along the $\mathrm{z}$ axis.

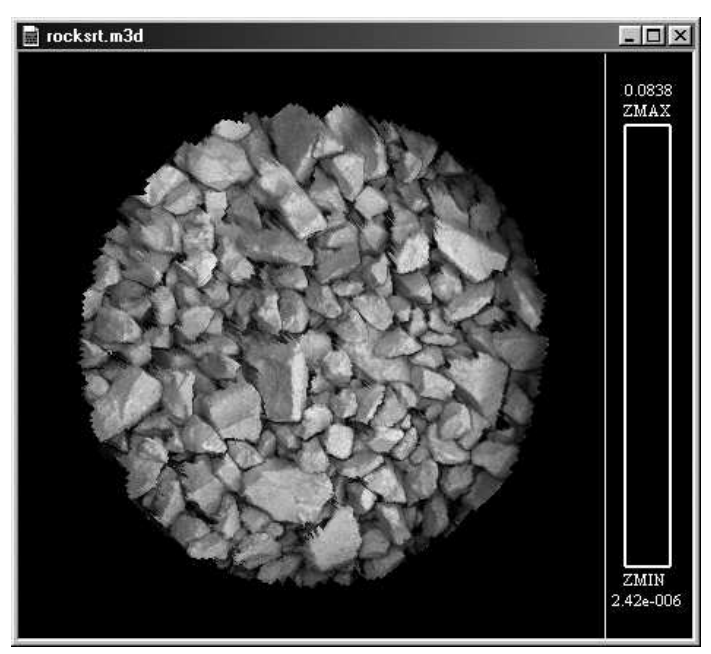

Fig. 2. Image overlay on the 3D surface model for the $020117 \mathrm{~b}$ measurement set

Each experiment was carried out in the following way.

Mixing The rock pile was mixed to homogenize the size classes within the pile.

Dumping The rocks were then dumped into a measurement bucket.

Marking The surface of the pile was sprayed with Flawcheck ${ }^{2}$ powder spray and then scanned with the Monash Shape Measurement system. All the visible fragments were marked with a colored marker to identify them as the surface fragments.

Counting Dominant visible fragments (those that were either entirely visible or had only a small edge or corner occluded) had their corresponding sieve

$\overline{2}$ Flawcheck step 4 developer evaporative powder spray. Dy-Mark (Aust.) Pty. Ltd. 89 Formation Street, Wacol, Qld, Australia 


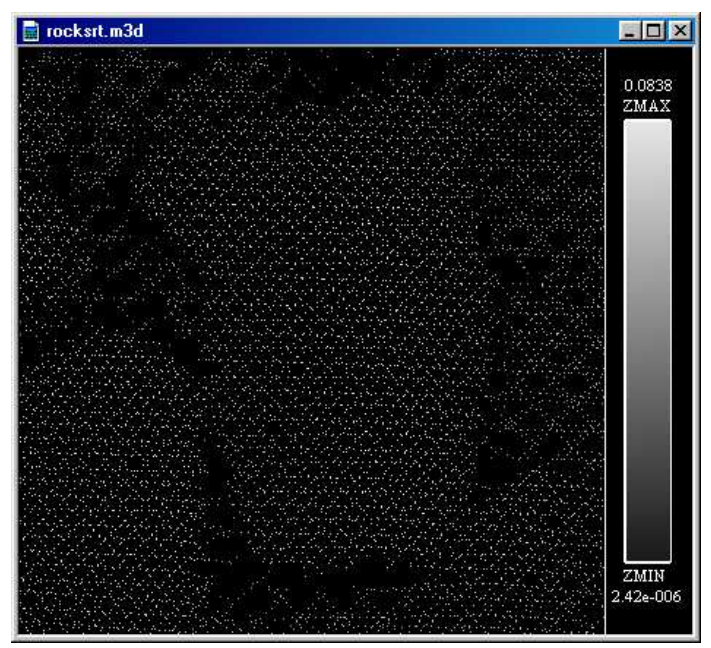

Fig. 3. 3D coordinate data of the central large fragment for the 020117b measurement set

size recorded on print-outs of the six camera images of the rock pile. All of the marked rocks were then counted and categorized according to sieve size and washed to remove the powder spray. The proportions of observed fragments by sieve size is shown in table 2 .

Remixing The removed surface rocks were remixed with the remaining contents of the bucket.

Note that the sieve size of any fragment is immediately known as the fragments have all been color coded according to sieve size as shown in table 1.

The results of 10 experiments are presented with each yielding three sets of results:

S.1 a set of 3D surface data of the rock pile surface built up from scans from six different viewing angles,

S.2 a set of observed counts (table 2) indicating the actual number of rocks of each size class on the surface of the pile, and

S.3 a collection of six printed images of the surface of the rock pile marked with the location and sieve size of the dominant visible fragments.

The printed camera images noted in S.3 define a map that has been manually entered onto the 3D data of each measurement using the $3 \mathrm{D}$ visualization software developed in this research. This map allows the comparison of individual fragments in the segmentation results with the corresponding surface rock fragments of known sieve size. 
Table 2

Counts of visible surface rocks by sieve size

\begin{tabular}{|lcccccccc|}
\hline test ID & \multicolumn{3}{c}{ Dominant Rocks $\dagger$} & \multicolumn{5}{c|}{ All Rocks } \\
yymmdd & pink & white & yellow & total & pink & white & yellow & total \\
\hline 020117a & 84 & 28 & 11 & 123 & 149 & 55 & 18 & 222 \\
020117b & 59 & 35 & 13 & 107 & 115 & 57 & 21 & 193 \\
020118b & 84 & 29 & 13 & 126 & 141 & 59 & 19 & 219 \\
020123b & 46 & 27 & 13 & 86 & 118 & 56 & 22 & 196 \\
020124a & 49 & 37 & 15 & 101 & 112 & 64 & 24 & 200 \\
020124b & 66 & 25 & 10 & 101 & 140 & 48 & 21 & 209 \\
020125a & 79 & 31 & 6 & 116 & 170 & 52 & 12 & 234 \\
020125b & 64 & 30 & 13 & 107 & 128 & 53 & 21 & 202 \\
020125c & 60 & 23 & 18 & 101 & 116 & 47 & 26 & 189 \\
020128a & 71 & 34 & 9 & 114 & 128 & 59 & 20 & 207 \\
\hline mean & 66.2 & 29.9 & 12.1 & 108.2 & 131.7 & 55.0 & 20.4 & 207.1 \\
\hline
\end{tabular}

$\dagger \quad$ Dominant rocks - Fragments that were either entirely visible or had only a small edge or corner occluded.

\section{A Discussion on Interpolating to a Regular Grid}

One possibility for processing the 3D data is to interpolate it to a regular image space in order to use existing morphological algorithms. However interpolation has several limitations that we shall examine here.

In general, interpolating to a regular grid means resampling the data at a sufficiently high density such that the detail and integrity of the data is maintained. As a side effect, this also means introducing additional data points, and as a result, various erroneous "artifacts" into the resultant data.

For data that has a non-uniform density, such as the data collected in this research where we have four times as many data points along the stripes as across the stripes, interpolation means either introducing a large number of additional data points, or discarding data.

The 3D data used here contains areas of occlusion, being areas of no data, which are particularly useful for determining fragment edges. By resampling to a regular grid we fill in these occluded areas by interpolating between neighbour points. This can create homogeneous areas in the data and bridges 
that join fragments. Bridge artifacts are of particular concern as they cause merging problems for techniques that use geodesic dilations such as watershed segmentation and morphological reconstruction.

Mathematical morphological algorithms are currently applied to 3D data in the form of greyscale images where the intensity represents the $\mathrm{z}$ depth dimension. Interpolation to a greyscale image space has further limitations in that the $\mathrm{z}$ depth resolution of the data is limited to a discrete depth range, typically 256 distinct levels ( 8 bits), although this is less significant if 16 or 24 bit greyscale depth is available.

Furthermore, mathematical morphology had not previously been applied to irregularly spaced 3D coordinate data. Such a capability suggested several advantages:

- to overcome the need to re-sample and thus prevent the introduction of erroneous artifacts into the data,

- to allow double precision for the depth instead of the typical 8 bit depth of a greyscale image space.

Based on these considerations a 3D solution was developed.

\section{Mathematical Morphology for 3D Surface Data}

In this section we introduce the field of mathematical morphology and provide definitions for applying mathematical morphology to irregularly spaced 3D coordinate surface data.

Mathematical morphology is a theory of transformations for the analysis of spatial structure in image data. It is derived from set theory and was initially developed by Matheron [31]. These transformations can be used to decompose input data into component features, identifying the underlying shapes. Serra $[32,33]$ provides a thorough introduction to mathematical morphology for two dimensional binary image sets while Serra [32] and Sternberg [34] detail the extension of mathematical morphology to greyscale image sets. In greyscale morphology we consider a greyscale image as an intensity depth map representing a three dimensional undulating surface across a regular grid. Sternberg [34] conceptualized greyscale mathematical morphology by equating greyscale images to "three dimensional surfaces whose topology is modified by sliding geometric structures" (or structuring elements). Serra [32] defined the umbra transform as the volume under the greyscale image surface upon which morphological transforms using three dimensional volumetric structuring elements could be applied. 
This three dimensional interpretation of greyscale morphology for image sets is readily extendable to the surfaces of irregularly spaced $3 \mathrm{D}$ coordinate points collected in this research. We develop a framework of mathematical morphological transformations in section 6.1 for application to this data and build from this a library of morphological operations.

The reader unfamiliar in mathematical morphology may refer to any of the cited publications or to Haralick and Shapiro [35, ch. 5] which provides a particularly clear and readily accessible presentation of binary and greyscale mathematical morphology.

As a general overview we may conceptualise erosion and dilation in terms of the undulating surface of the rock pile, as defined by the 3D coordinate data. We can dilate the rock pile surface by rolling a structuring element sphere over every part of the rock pile surface. If we track the center of the sphere as it rolls over the surface we define a new surface set corresponding to the dilation of the rock pile surface using the spherical structuring element. Similarly, we can define erosion by rolling the sphere across the underside of the pile surface, tracking the center of the sphere to define the new eroded surface set.

\subsection{Defining Erosion and Dilation}

In this section we define morphological operations derived from greyscale morphology for use with irregularly spaced 3D coordinate data.

A greyscale image is well constrained and relatively simple to apply morphological operations to. It is a discrete space with well defined spatial relationships between points and their neighbours as they are equally spaced on a regular grid. The 3D irregularly spaced coordinate points used in this research are not defined in a regular space and as a result we cannot use discretely specified structuring elements. We must use continuous functional structuring elements so that the structuring element is defined for the neighbourhood of any element of the data.

Let $S_{i}=\left\{(x, y, z) \in R^{3}\right\}$ represent the 3D coordinate data from a single scan of a scene using the Monash Shape Measurement system. Let the set $S=\bigcup_{i} S_{i}$ represent a set comprising multiple scans of this scene using the same coordinate system so that all of these views are registered. The set $S$ does not have the features of well defined spatial relationships between neighbours, simple transformation to a volume, or ease of applying the structuring element associated with a greyscale image space.

We conceptualize $S$ as a set of surface points partially defining the surface of an unknown volume and define erosion and dilation as it relates to these known 
surface data points. We conceptualize the structuring element as a volume whose surface is defined by the structuring element function. We suitably orientate the set with respect to the $\mathrm{z}$ axis as greyscale morphological erosion represents a series of negative translates in $\mathrm{z}$ and dilation represents a series of positive translates.

For erosion we find the maximum values of $\mathrm{z}$ for which we can fit the structuring element volume underneath the surface set $S$. As defined in Haralick and Shapiro [35, ch. 5], erosion and dilation are dual transformations with respect to complementation. We shall exploit this duality in order to define dilation. Therefore, for dilation we find the minimum values of $\mathrm{z}$ for which the negative transpose of the structuring element volume sits on top of the surface set $S$.

We now provide a definition for erosion and dilation suitable for use on irregularly spaced $3 \mathrm{D}$ coordinate data that partially defines the surface of an unknown volume. The mathematical definitions presented are extensions of the greyscale morphological definitions given in Haralick and Shapiro [35, ch. 5].

We define the discrete set $S$ in equation 1 representing the surface data set.

We define the surface function $f$ in equation 2 as a mapping from the domain to the range of $S$.

We define the structuring element function $k$ in equation 3 that defines a structuring element $B$ (equation 4 ).

We define the transpose structuring element function $\hat{k}$ and corresponding transpose structuring element $\hat{B}$ in equations 5 and 6 .

We then define $B_{p}$ in equation 7 as the translation of the set $B$ from the origin to the point $p$.

Finally we define the erosion of the surface function $f$ by the structuring element function $k$ in equation 8 and the dilation in equation 9 .

The Surface Data Set

$$
\begin{aligned}
S & =\left\{(x, y, z) \in R^{3}\right\} \\
F & =\{(x, y):(x, y, z) \in S\} \\
f & : F \rightarrow R, \text { where } f(x, y)=z \text { and }(x, y, z) \in S
\end{aligned}
$$

The Structuring Element

$$
\begin{aligned}
K & \subset R^{2} \\
k & : K \rightarrow R, \text { where } k(x, y)=z
\end{aligned}
$$




$$
\begin{aligned}
B & =\{(x, y, k(x, y))\} \\
\hat{k} & : K \rightarrow R, \text { where } \hat{k}(x, y)=k(-x,-y) \\
\hat{B} & =\{(x, y, k(-x,-y))\} \\
B_{p} & =\{p+b: p=(x, y, z), b \in B\}
\end{aligned}
$$

Erosion

$$
\begin{aligned}
(f \ominus k)(x, y) & =\max \left\{z: p=(x, y, z), B_{p} \leq f(a, b) \forall(a, b) \in F\right\} \\
& =\max \{z: z+k(a-x, b-y) \leq f(a, b) \forall(a, b) \in F\}
\end{aligned}
$$

Dilation

$$
\begin{aligned}
(f \oplus k)(x, y) & =\min \left\{z: p=(x, y, z), \quad(-\hat{B})_{p} \geq f(a, b) \forall(a, b) \in F\right\} \\
& =\min \{z: z-k(x-a, y-b) \geq f(a, b) \forall(a, b) \in F\}
\end{aligned}
$$

\subsection{Iterative Rule}

The iterative rule for erosion and dilation can be used to produce morphological transformations using larger and more complex structuring elements by successively applying simpler structuring elements.

The iterative rule does not hold for the morphological operations defined here due to the discrete and irregular nature of the data. As stated previously, the surface data is irregularly distributed in 3D and as a result we use continuous structuring elements that can be applied to any coordinate value within range. If we consider the iterative rule for dilation from binary morphology Haralick and Shapiro [35, Ch. 5] and define $S$ as the discrete 3D surface set, and $B$ and $C$ as continuous structuring element spheres of radius $\mathrm{r}$, we can demonstrate the inequality of the iterative rule in this case.

The iterative rule for dilation states $(S \oplus B) \oplus C=S \oplus(B \oplus C)$. From our definition of the structuring elements $B$ and $C$ we can conclude that $(B \oplus C)$ is a continuous sphere of radius $2 r$. If the radius $r$ is less than the distance between points in set $S$, then a dilation by $B$ is equal to the translation of set $S$ along the $z$ axis by the amount $r$. The dilation at each point in $S$ transforms only that point as no other points are in range. Two successive dilations will equal a translation by the amount $2 r$. If the radius $2 r$ is greater than the distance between points, then dilation by a sphere of radius $2 r$ at a point in $S$, will transform potentially several neighbouring points. Therefore, successive dilations by spheres of radius $r$ on set $S$ is not equivalent to dilation by the 
sphere of radius $2 r$. In more general terms, we can define that a dilation by a sphere of radius $r$ will on average transform $n$ points, and that a dilation by a sphere of radius $2 r$ will on average transform $m$ points. Depending on the distance between points in $S$ we have shown that $m>2 n$ and as a result the iterative rule does not hold.

We note however, that morphological structuring element decomposition can still be applied to perform operations using larger structuring elements based on a series of operations using smaller structuring elements. Given structuring elements $B$ and $C$, both with radius larger than the distance between neighbouring points, we can combine morphological operations and use the iterative rule to determine the size of the structuring element that would produce a similar result. However, it is not appropriate to use the iterative rule to determine what the equivalent larger structuring element would be.

\subsection{The Implemented Image Analysis Operations}

The morphological operations of erosion and dilation have been implemented for spheres and flat circular disk structuring elements. Using erosion and dilation as a basis we have implemented a library of morphological operations encompassing, opening, closing, morphological gradient from Beucher and Meyer [36, pg. 435], morphological top hat from Beucher and Meyer [36, pg. 435], morphological reconstruction from Serra and Vincent [37, pp. 72-75], morphological decomposition from Pitas and Maglara [38], and finally watershed segmentation from Beucher and Meyer [36]. In addition the following supporting operations have been implemented, median filter, thresholding, adaptive thresholding, distance transform from Borgefors [39], and region edge following.

The library of code has been implemented in $\mathrm{GNU} \mathrm{C} / \mathrm{C}++$ and $\mathrm{C}$ shell under GNU Linux. At the heart of the implementation is the 3D data structure allowing relatively fast 3D lookup of arbitrary cubic volumes. A flexible two or three dimensional kd-tree has been implemented for this purpose from the algorithm provided in de Berg [40, section 5.2].

\subsection{D Image Analysis Performance}

Timing measurements were performed on an PC running linux with an Intel Celeron $850 \mathrm{MHz}$ CPU. Executables were compiled using the gnu c++ compiler version 3.2 with 03 optimisation.

The 020117a measurement was used comprising 150511 3D coordinate points 
of the rock pile surface within a circular bucket of diameter $265 \mathrm{~mm}$. On average there are approximately 2.73 points per square millimeter when the data is projected into the $\mathrm{x}-\mathrm{y}$ plane.

Table 3 shows the execution times for the base morphological operations, and table 4 shows the execution times for the segmentation operations defined in section 7 .

Table 3

\begin{tabular}{|lccr|}
\hline Operation & $\begin{array}{c}\text { Structuring } \\
\text { Element }\end{array}$ & $\begin{array}{c}\text { Radius } \\
(\mathrm{mm})\end{array}$ & $\begin{array}{r}\text { Time } \\
(\mathrm{sec} .)\end{array}$ \\
\hline \multicolumn{3}{|c}{ Base Operations } \\
erosion or dilation & sphere & 3 & 20.3 \\
erosion or dilation & sphere & 10 & 142.0 \\
opening or closing & sphere & 3 & 35.9 \\
opening or closing & sphere & 10 & 282.5 \\
\hline
\end{tabular}

Execution times for the base morphological operations on measurement 020117a

\subsection{Defining the Local Neighbourhood}

For many of the operations implemented it is necessary to examine the neighbours of any given point in the data. However, as we are dealing with multiple overlapping scans of data we do not have an existing relationship between individual points and all of their neighbours. Within a single scan we are aware of the neighbour points using the column and row information derived from the camera images of the light stripes, but we must search for points within a specified spatial domain to determine the spatial neighbours from all scans for a given point.

As previously described the 3D model contains six overlapping sets of data collected at rotations of $0,45,135,180,225$, and 315 degrees. We can exploit this overlapping structure to define a local neighbourhood radius based on the spacing between the neighbouring points in the rows of a single scan. We do not need to ensure a local neighbourhood radius large enough to identify neighbouring points in the columns (between the rows), as the rows of data from a single scan are nominally connected to each other by the overlapping rows of data from four of the remaining five scans, the fifth scan containing effectively parallel rows of data as for each scan there is another scan collected at +180 degrees. As a result we define the local neighbourhood radius based on our knowledge of the spacing calculated from the individual scans, even though we will be processing them in combination. 


\begin{tabular}{|c|c|c|c|}
\hline Operation & $\begin{array}{c}\text { Structuring } \\
\text { Element }\end{array}$ & $\begin{array}{c}\text { Radius } \\
\text { (mm) }\end{array}$ & $\begin{array}{r}\text { Time } \\
\text { (sec.) }\end{array}$ \\
\hline \multicolumn{4}{|c|}{ Segmentation Operations $\dagger$} \\
\hline closing & sphere & 10 & 283.8 \\
\hline subtraction & - & - & 7.7 \\
\hline depth threshold & - & $5 \%$ & 3.0 \\
\hline mask & - & - & 6.9 \\
\hline distance transform & - & 20 & 18.8 \\
\hline opening & sphere & 3 & 42.3 \\
\hline reconstruction $\ddagger$ & circle & 3 & 230.2 \\
\hline mask & - & - & 6.4 \\
\hline seeds & - & - & 54.3 \\
\hline closing (of original) & sphere & 3 & 36.1 \\
\hline watershed & circle & 1 & 14.8 \\
\hline seg filter & circle & 2 & 12.1 \\
\hline entire segmentation & - & - & 755.7 \\
\hline $\begin{array}{l}\text { Segmentatior } \\
\text { utively. }\end{array}$ & operations are & erformec & consec- \\
\hline Required 11 & eodesic dilation & {$[37, \mathrm{pp}$.} & 2]. \\
\hline
\end{tabular}

Table 4

Execution times for the segmentation of measurement 020117b

We note that the rock pile surface is aligned in the $\mathrm{x}-\mathrm{y}$ plane and that depth variation in the pile surface corresponds to variations along the $\mathrm{z}$ axis. If we consider a row of data corresponding to one of the light stripes from a single scan, we can analyse the $\mathrm{x}-\mathrm{y}$ distance between points with neighbouring column numbers, being points derived from neighbouring pixels in the camera image. This analysis results in the determination that the mean distance between neighbouring points in the rows of data for the 020117a data set, is approximately $0.68 \mathrm{~mm}$ with a standard deviation of approximately $0.1 \mathrm{~mm}$. In addition we determine that over $99 \%$ of the data points from all six scans are closer to their immediate row neighbours than the mean plus three standard deviations. Therefore we define the local neighbourhood of any given point as the points within a $1 \mathrm{~mm}$ radius circle as we are certain that more than $99 \%$ of the data points have immediate neighbours from the same scan within this range, and are also likely to have points from other scans within this range. 


\section{Segmenting the $3 \mathrm{D}$ Coordinate Surface Data}

We now segment the 3D data sets in order to identify all of the surface fragments. The presented technique uses the watershed segmentation approach of Beucher and Meyer [36] which requires the identification of suitable seed regions as input. We shall identify these seed regions using a combination of edge detection techniques, distance transform and local maxima detection.

Each of the ten data sets were processed using the following sequence of morphological and mathematical operations outlined in detail in the following sections.

\section{Segmentation Process}

7.1 Median filter

7.2 Detecting the edges of occluded areas

7.3 Morphological edge detection

7.4 Combining edge detection techniques

7.5 Distance transform

7.6 Calculating the local maxima

7.7 Forming seed regions

7.8 Watershed segmentation

7.9 Filtering

7.10 Defining the accurate segmentation for comparison

7.11 Quantifying the calculated segmentation

\subsection{Median Filter}

A median filter was performed on each data set to remove slight noise variation particularly at the $(\mathrm{x}, \mathrm{y})$ boundaries of the data and between data points from different scans.

The median filter was performed as follows: determine a $1 \mathrm{~mm}$ radius neighbourhood in the $\mathrm{x}, \mathrm{y}$ plane about a given point 'p' defining a cylindrical volume along the $\mathrm{z}$ axis, compare all of the points within this neighbourhood by $\mathrm{z}$ depth, calculate the median $\mathrm{z}$ depth value and assign the $\mathrm{z}$ depth of the given point 'p', to this median value.

Figure 4 shows the absolute values of the largest $95 \%$ of $\mathrm{z}$ values subtracted out by this filtering process for the $020117 \mathrm{~b}$ measurement set. 


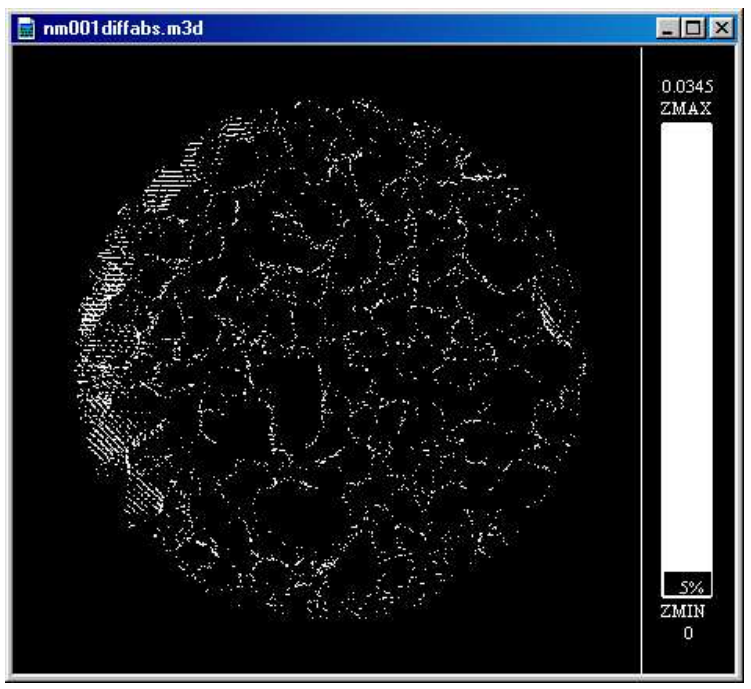

Fig. 4. Data filtered out of the $020117 \mathrm{~b}$ measurement set using a $1 \mathrm{~mm}$ radius median filter. The absolute value of the largest $95 \%$ of $\mathrm{z}$ values is shown.

\subsection{Detecting the Edges of Occluded Areas}

As the data has been collected using a camera offset from the light stripe projector, as shown in figure 1, each scan contains areas of occlusion where the stripes from the projector are not visible from the camera's view point. These occluded areas correspond to edges in the data, primarily on the boundaries between fragments, but occasionally at sharp ridges near the apex of fragments. These edges of occluded areas are detected by a discontinuity in the camera pixel number along the projected stripe. Figure 5 shows the $3 \mathrm{D}$ coordinate points of the line neighbours of occluded points, combined for all six views of measurement set $020117 \mathrm{~b}$. We define these coordinates as the 'edges of occluded areas' for the given measurement.

\subsection{Performing Morphological Edge Detection}

The occluded edge set can contain edge points corresponding to abrupt ridge lines on fragments that need to be removed. By using a second method of edge detection we achieve this goal.

Typically a morphological gradient transform from Beucher and Meyer [36, pg. 435] is performed to detect edges. The morphological gradient is calculated by subtracting the result of an erosion, which erodes the peaks in the data, from the result of a dilation, which fills in the valleys in the data set. Since we are not interested in detecting both the peaks and the valleys using a morphological edge detection the morphological gradient operation is not suitable. 


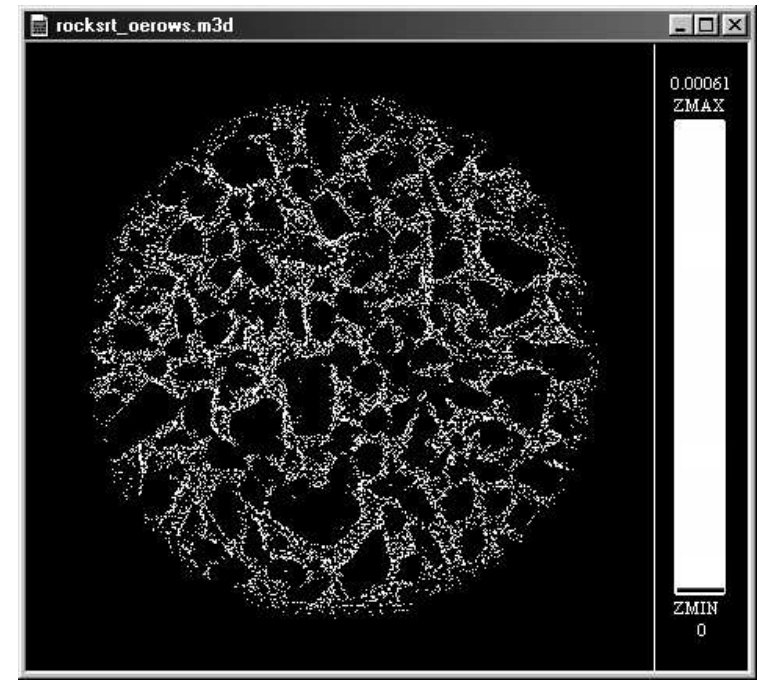

Fig. 5. Edges of occluded areas for the 020117b measurement set

We perform morphological edge detection by applying the black top hat transform from Beucher and Meyer [36, pg. 436] to subtract the data set from its morphological closing. The black top hat predominantly detects the valleys in the data, which will identify the boundaries between fragments and not peaks on the fragments. We shall use the results from the black top hat transform to confirm which edges in the occluded edge set we wish to keep.

Detecting edges using combinations of morphological erosions and dilations requires the selection of an appropriate structuring element. The morphological operation is then followed by a thresholding operation that separates the detected features into edges and non-edges. There is significant room for variation in the combination of these two operations. A larger structuring element increases the proportion and strength of the edges detected. However, it typically ensures that after thresholding, fragments smaller than the structuring element are entirely included in the subset of edges. If the structuring element is too small then insufficient edges are detected to distinguish between neighbouring fragments. A balance must be determined between these user variable factors to calculate the edges appropriate for a given application.

A black top hat operation using a large $10 \mathrm{~mm}$ radius spherical structuring element was performed to strongly emphasize the boundaries between fragments. A five percent depth threshold was then applied to the black top hat data, reducing the smallest five percent of $\mathrm{z}$ coordinate data to zero. The resultant thresholded data set (for the 020117b measurement) is shown in figure 6 .

The choice of a five percent threshold for the black top hat morphological edge set is flexible but it is important that the threshold is kept low. A higher threshold leads to an overly sparse morphological edge set which leads to too few edges of occluded areas being kept for further analysis. 


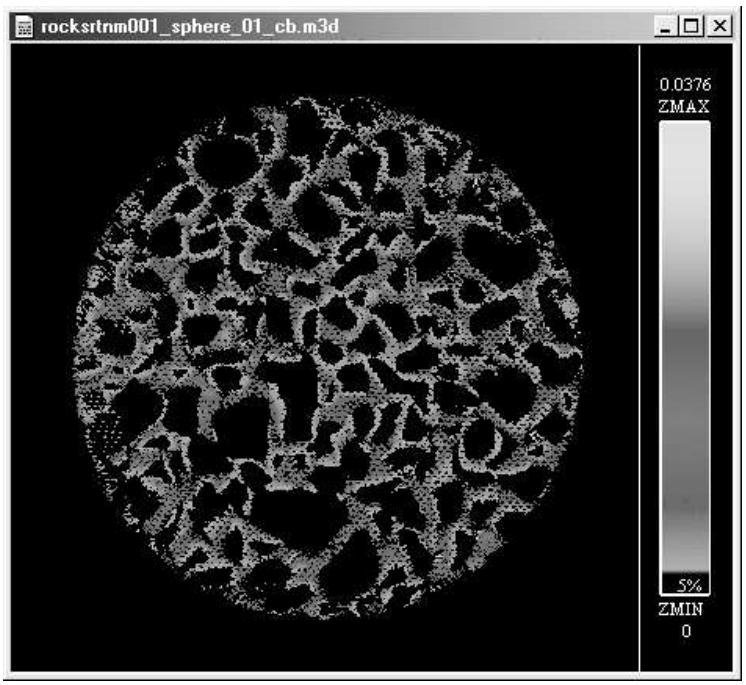

Fig. 6. Edge detection using the black top hat operator with a $10 \mathrm{~mm}$ radius structuring element and $5 \%$ threshold on $\mathrm{z}$ depth on the $020117 \mathrm{~b}$ measurement set

\subsection{Combining the Edge Detection Techniques}

The edges of occluded areas described in section 7.2 are detected by the measurement system and require no user variable operations such as those described for morphological edge detection techniques. We therefore use the edges of occluded areas as the basis of the edge detection presented with the morphological technique used to refine the results.

Both the occluded edge data and the black top hat data have been derived from the same data set and as such contain exactly the same $(\mathrm{x}, \mathrm{y})$ components of their 3D coordinate points. We shall use the thresholded black top hat data set, based on zero values of $\mathrm{z}$ (not an edge), to mask the corresponding $(\mathrm{x}, \mathrm{y})$ points in the occluded edge data set to zero. This operation reduces the number of edge points in the occluded edge set, specifically in locations near the centers of larger fragments.

Figure 7 shows the result of this operation. Without this operation some of the large prominent rock fragments would be over-segmented as multiple seeds would be formed for these fragments in the subsequent seed formation step.

\subsection{Calculating the Distance Transform}

The distance transform from Borgefors [39] is applied to the resultant edge data to determine the distance in the $\mathrm{x}, \mathrm{y}$ plane from any given point to the nearest edge point (a coordinate point with non-zero $\mathrm{z}$ in the edge data). The distance values are stored in the $\mathrm{z}$ value of each coordinate point and the 


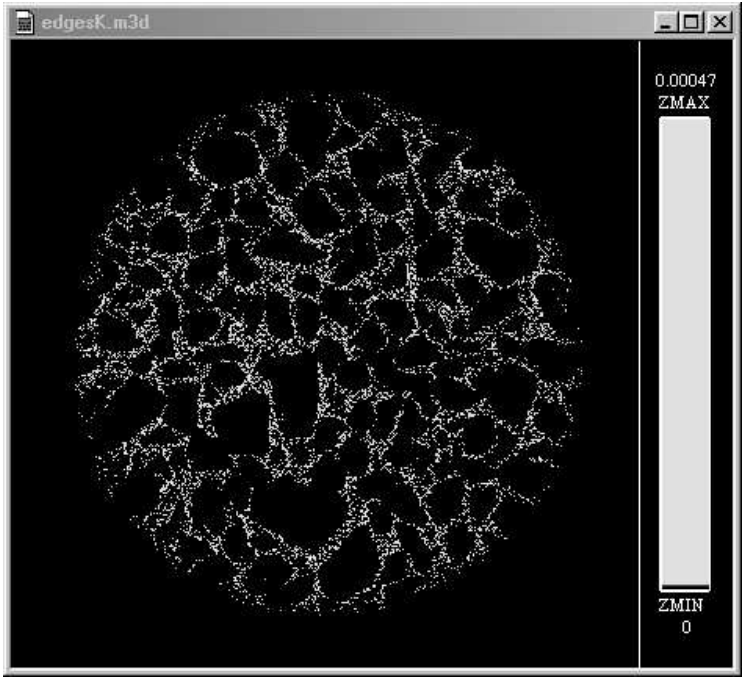

Fig. 7. Detected fragments edges for the $020117 \mathrm{~b}$ measurement set

results for the $020117 \mathrm{~b}$ measurement set are shown in figure 8 . The peaks in the distance transform will form the basis for specifying the seed regions for input into the watershed segmentation.

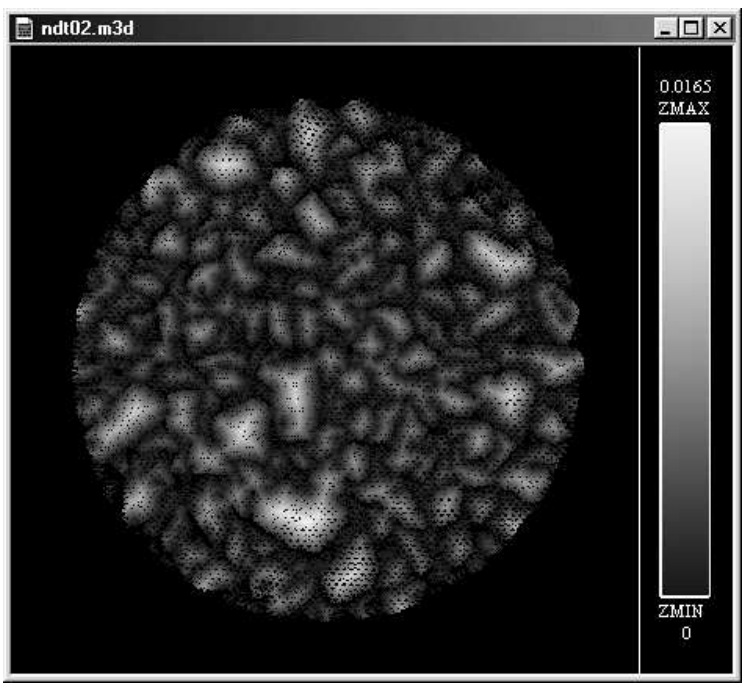

Fig. 8. Distance transform applied to the final edges data shown in figure 7 showing the distance to the nearest fragment edge

\subsection{Detecting the Peaks in the Distance Transform}

We apply the technique of opening by reconstruction from Serra and Vincent $[37$, pp. $72-75]$, to detect the local maxima in the distance transform results.

The following three step process performs the opening by reconstruction and detects the local maxima: 
(1) an opening is performed on the distance transform data using a spherical structuring element of radius $3 \mathrm{~mm}$,

(2) successive geodesic dilations outlined in Serra and Vincent [37, pp. 72] are performed using a $3 \mathrm{~mm}$ radius circular disk structuring element, and

(3) the local maxima set is determined by subtracting the reconstructed result from the input distance transform data.

We note that the definition of geodesic dilations, as applied to reconstruction, provided by Serra and Vincent [37], calls for a unit sized structuring element. In the context of the morphological definitions provided for irregular 3D data we do not have a clear definition of 'unit' size, such as one pixel in binary and greyscale images. Based on the definition provided for the local neighbourhood radius in section 6.5 we could use a definition of $1 \mathrm{~mm}$. A larger geodesic dilation radius increases the possibility of inappropriately reconstructing, and therefore not detecting, spatially close but distinct maxima. In the reconstruction performed here we have applied $3 \mathrm{~mm}$ radius geodesic dilations as it provided a substantially quicker computation without any significant reduction in detected maxima.

The local maxima determined by this operation are then masked with the distance transform data such that zero $\mathrm{z}$ values in the local maxima data, mask the $\mathrm{z}$ values of corresponding $(\mathrm{x}, \mathrm{y})$ pairs in the distance transform data to zero. The resultant data set is shown in figure 9 and represents the local maxima data with each $\mathrm{z}$ value indicating the distance to the nearest edge point in the $\mathrm{x}, \mathrm{y}$ plane.

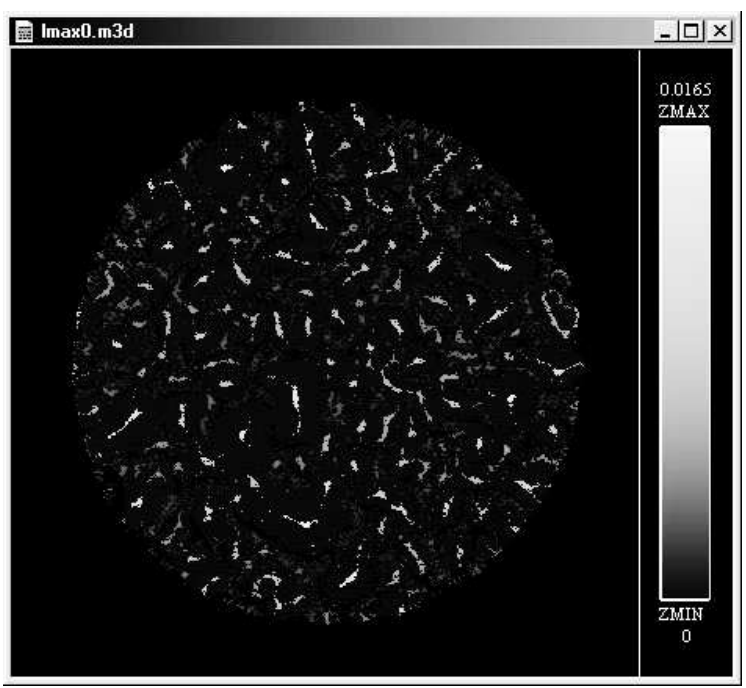

Fig. 9. Local maxima of the distance transform data set shown in figure 8 showing the distance to the nearest fragment edge 


\subsection{Calculating the Seed Regions}

The local maxima results are used to form seed regions for input into the watershed segmentation algorithm. The seed regions are formed using the following process which is detailed with the aid of figure 10:

(1) The local maxima points shown in figure 9 are sorted from highest $\mathrm{z}$ value, being the point furthest from an edge, down to the lowest $\mathrm{z}$ value. Note that the points in figure 10 are ordered $a, b, c$ from highest $\mathrm{z}$ to lowest $\mathrm{z}$ value.

(2) For each local maximum point beginning with the point of highest $\mathrm{z}$ value:

- Create a seed circle centered at the $(\mathrm{x}, \mathrm{y})$ of the current maximum point, with diameter equal to its $\mathrm{z}$ value. The seed circle encompasses all of the data points (maxima and non-maxima points) within its perimeter. Figure 10 shows the shaded seed circle formed for point $a$ of diameter $a_{z}$ where $a=\left(a_{x}, a_{y}, a_{z}\right)$.

Exception 1 If the (x,y) location of the current point is within the bounds of an already formed seed region, ignore the current point and its seed circle. This exception prevents a runaway merging process in which only one giant seed region is formed. Point $b$ in figure 10 is within the existing seed region $R_{a}$ and is therefore ignored.

Exception 2 If the current seed circle overlaps an existing seed region, merge the seed circle into that region. This exception ensures that long rectangular like seed regions can be built up from multiple seeds. The seed circle around point $c$ in figure 10 intersects with seed region $R_{a}$ and will therefore be merged into $R_{a}$.

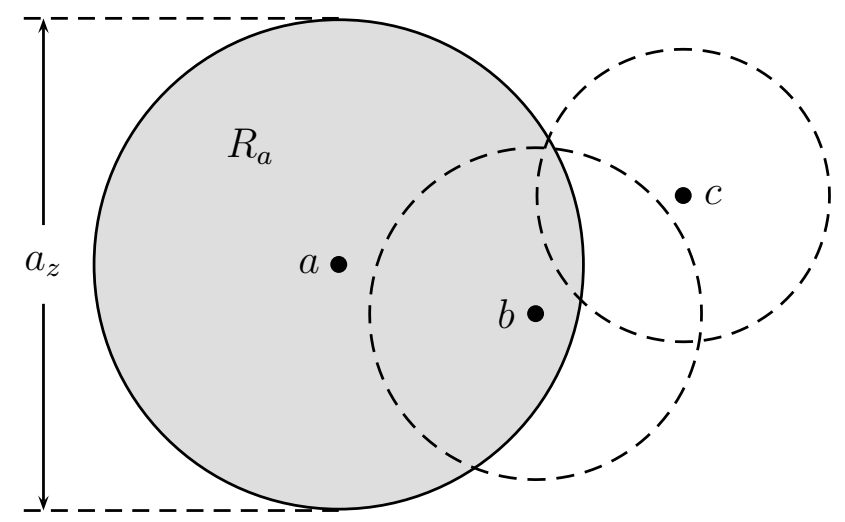

Fig. 10. Creating seed region $R_{a}$ by merging seed circles built up from distance transform local maxima points $a, b$ and $c$.

Using this process 391 seed regions are formed for the $020117 \mathrm{~b}$ measurement set as shown in figure 14 (page 32). 


\subsection{Performing the Watershed Segmentation}

The watershed segmentation algorithm from Beucher and Meyer [36] has been implemented for 3D coordinate surface data. The reader is referred to the afore mentioned publication for a detailed explanation of this technique. Only a simplified summary of the technique is provided here.

The watershed algorithm is applied to an edge set, typically produced using the morphological gradient operator. As mentioned in step 7.3 the black top hat operation forms an edge set more suited to the identification of the boundaries between rock fragments. Therefore a black top hat set has been generated using a $3 \mathrm{~mm}$ radius spherical structuring element. Figure 11 shows the resultant black top hat for the 020117b measurement set.

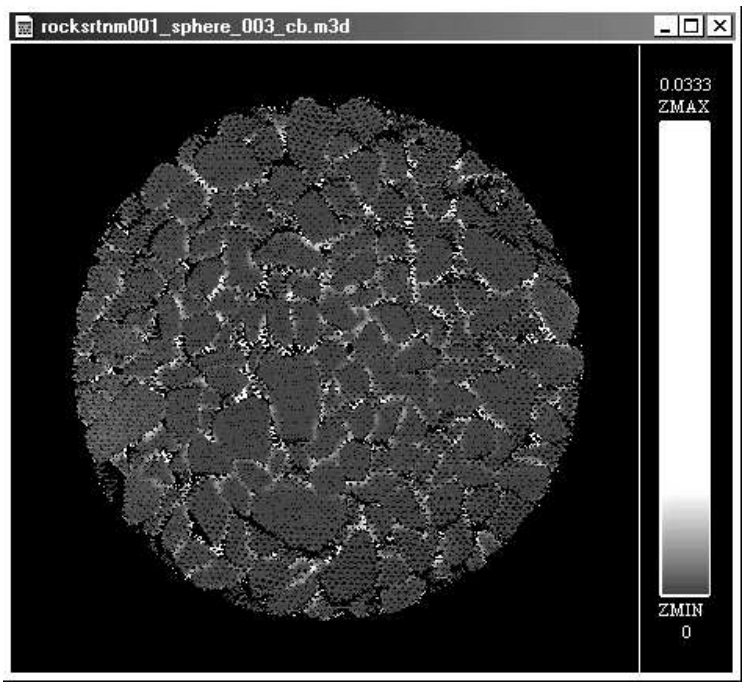

Fig. 11. Fragment edges detected using the black top hat morphological operation using a $3 \mathrm{~mm}$ radius spherical structuring element. Applied to the median filtered 020117b measurement set.

The watershed approach considers the input edge set as an undulating terrain map. Much of the terrain map consists of flat regions corresponding to the rock fragments surrounded by ridge lines corresponding to the edges and boundaries between fragments. The watershed algorithm floods the terrain map by progressively increasing the watertable. Pools of water form in minima in the terrain and define unique regions that expand across the terrain as the watertable is increased. Boundaries are formed between regions when the increasing watertable would otherwise cause them to join, similar to a dam wall built between two neighbouring and rising pools. However, the simple watershed algorithm outlined suffers from over-segmentation.

We enhance the watershed segmentation using flooding from selected sources described by Beucher and Meyer [36, pg. 447] with the selected sources being 
the seed regions calculated in section 7.7. The identified seed regions form wells in the undulating terrain of the black top hat edge set and define unique regions. The watertable depth is increased using the seed wells as water sources. Each coordinate point is analysed as the watertable meets the depth of its component $\mathrm{z}$ value. An analysed point is only added to a region if it is within $1 \mathrm{~mm}$ in the $\mathrm{x}-\mathrm{y}$ plane of points already within a flooded region. When the watertable increases such that it would flow over a ridge in the edge data and into a valley not already filled with water (classified as already belonging to a region), the water flows out into the valley using successive $1 \mathrm{~mm}$ geodesic dilations from Serra and Vincent [37, pp. 72].

\subsection{Filtering the Resultant Segmentation}

The segmentation resulting from the watershed algorithm contains as many regions as the number of input seed regions. Many of them are very small and clearly not representative of a rock fragment. For the $020117 \mathrm{~b}$ measurement set there are 391 regions in the segmentation and only 193 rocks manually counted as on the surface.

We perform a filtering step here to remove overly small regions. A morphological approach was considered as a morphological closing operation is ideally suited to removing small features. However, such an approach has problems in the irregularly spaced 3D data used here. Areas of occlusion with missing data points exist within the data set and many of the small regions exist next to these occlusions, or are at the circular $x-y$ plane boundaries of the data. A morphological closing approach with a small structuring element would not remove tiny regions that exist next to missing data points as the region will effectively be considered to be as large as the combination of the region and the expanse of missing data points.

The filtering method used calculates the maximum number of points that can fit within a circle of specified radius across the data set and removes all regions that have equal or fewer points. A radius of $2 \mathrm{~mm}$ has been used and for the 020117b measurement set the number of regions is reduced from 391 to 227 . We note however, that this is still larger than the 193 rocks manually counted on the surface of the $020117 \mathrm{~b}$ pile. The choice of the filtering radius is clearly somewhat arbitrary and could be more carefully tailored to match the known results. A radius of $3 \mathrm{~mm}$ removes too many regions and the $2 \mathrm{~mm}$ radius produces acceptable results. Filtering based on the possibility of merging overly small regions was also investigated but failed to produce results any better than the presented removal method.

The filtering technique applied here is very similar to the morphological tech- 
nique of area openings. According to Vincent [41], a "filter that removes from a binary image its connected components with area smaller than a parameter $\lambda$ is called area opening". In a binary image space the area of a connected component is defined by the number of pixels it contains. In this section we remove regions if they contain a number of data points less than a given threshold. This is similar, although not the same as an area opening as the number of data points is only an approximation of the area of a region.

Figure 15 (page 32) shows the result of the watershed segmentation after filtering for the $020117 \mathrm{~b}$ measurement set. Note that the presented image has had the boundaries of each region eroded by $1.5 \mathrm{~mm}$ so that the segmentation can be visually interpreted more readily.

\subsection{Defining an Accurate Segmentation for Comparison}

The 10 measurement sets presented in table 2 each contain a manually specified segmentation of the surface fragmentation of the pile. Each manually specified segmentation records all of the fragments visible in the images of the surface of the pile.

We use the manually specified segmentation to define the "accurate" segmentation. The manually specified segmentation contains numerous individually specified regions across the data set but it is not complete. There are points within the data set that are not included within a region as it can be difficult to tell from the images exactly where the boundary of a fragment is. When the boundary has been unclear we have pulled back the manually specified region by defining it short of the boundary. In addition, as we seek to quantify the results of a computed watershed segmentation, it is appropriate to compare it to other watershed segmentations. Therefore we define the "accurate" segmentation by performing a watershed segmentation using the manually specified segmentation as input seeds. The effect of this watershed segmentation is to expand the manually specified regions such that they border each other completely. Figure 13 (page 32) shows the final "accurate" segmentation for the 020117b measurement set.

\subsection{Quantifying the Calculated Segmentation}

To quantify the calculated segmentation we compare it to the "accurate" segmentation defined in section 7.10. From a visual comparison of figures 13 and 15 (both on page 32) there appears to be a strong correspondence between segmentations. We shall quantify the segmentation results by focusing on how many fragments have been over-segmented, and how many have been 
under-segmented. Over-segmentation occurs when a fragment region in the "accurate" segmentation is represented by multiple regions in the calculated segmentation, and under-segmentation occurs when multiple fragments in the "accurate" segmentation are identified by one region in the calculated segmentation.

Using this criterion, a segmentation free from under-segmentation and oversegmentation would contain the correct number of segmented regions, and all of these regions would have one primary match between the "accurate" and calculated segmentations. Such a segmentation is not a perfect segmentation as it allows variation in region size. However, this size variation will not be overly large, as by definition, primary matching regions contain the most number of common coordinate points.

In order to establish how much over-segmentation and under-segmentation has occurred, we determine, for each region, the corresponding region in the other segmentation that is its primary match. The primary match is the region that shares the most number of coordinate points. We then record all instances where multiple regions from one segmentation form a primary match with a single region in the other segmentation. Table 5 records the number of under and over-segmented regions, firstly for the dominant visible fragments, and then for all of the identified regions. Based on table 5 we note that $17.7 \%$ of the dominant visible regions are either under or over-segmented, and considering all of the segmented regions, $23.3 \%$ are either over or under-segmented. 
Table 5

Number of matching, under-segmented and over-segmented regions between the "accurate" and calculated segmentations

\begin{tabular}{|lcccccccccc|}
\hline test ID & \multicolumn{4}{c}{ Dominant Regions $\dagger$} & \multicolumn{5}{c|}{ All Regions } \\
yymmdd & mch & useg & oseg & total & \% err & mch & useg & oseg & total & \% err \\
\hline 020117a & 122 & 5 & 23 & 150 & 18.7 & 194 & 22 & 41 & 257 & 24.5 \\
020117b & 104 & 5 & 15 & 124 & 16.1 & 169 & 16 & 28 & 213 & 20.7 \\
020118b & 115 & 9 & 20 & 144 & 20.1 & 164 & 26 & 30 & 220 & 25.5 \\
020123b & 84 & 2 & 19 & 105 & 20.0 & 163 & 13 & 42 & 218 & 25.2 \\
020124a & 101 & 7 & 22 & 130 & 22.3 & 165 & 17 & 32 & 214 & 22.9 \\
020124b & 96 & 5 & 15 & 116 & 17.2 & 166 & 21 & 36 & 223 & 25.6 \\
020125a & 114 & 3 & 14 & 131 & 13.0 & 191 & 15 & 38 & 244 & 21.7 \\
020125b & 99 & 5 & 15 & 119 & 16.8 & 167 & 17 & 30 & 214 & 22.0 \\
020125c & 102 & 2 & 17 & 121 & 15.7 & 168 & 20 & 30 & 218 & 22.9 \\
020128a & 112 & 4 & 18 & 134 & 16.4 & 183 & 19 & 34 & 236 & 22.5 \\
\hline mean & & & & & 17.7 & & & & & 23.3 \\
\hline
\end{tabular}

$\dagger \quad$ Dominant regions - regions corresponding to the dominant fragments (either entirely visible or had only a small edge or corner occluded).

mch Number of correctly matching regions, for which there is only one primary match between the "accurate" and calculated regions.

useg Number of under-segmented regions in the calculated segmentation.

oseg Number of regions in the "accurate" segmentation that are over-segmented in the calculated segmentation.

$\%$ err Number of under-segmented and over-segmented regions as a percentage of the total number of regions. 


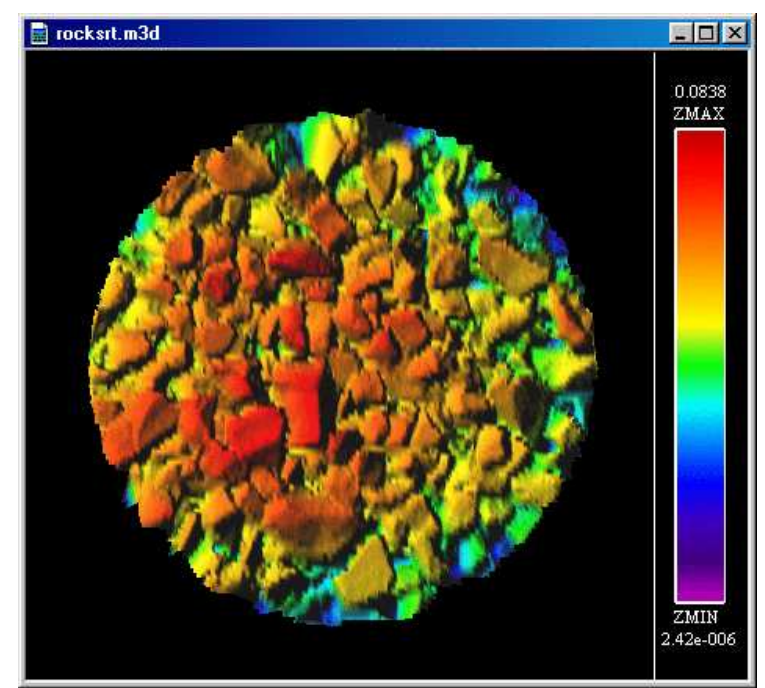

Fig. 12. 3D surface model of the 020117b measurement set

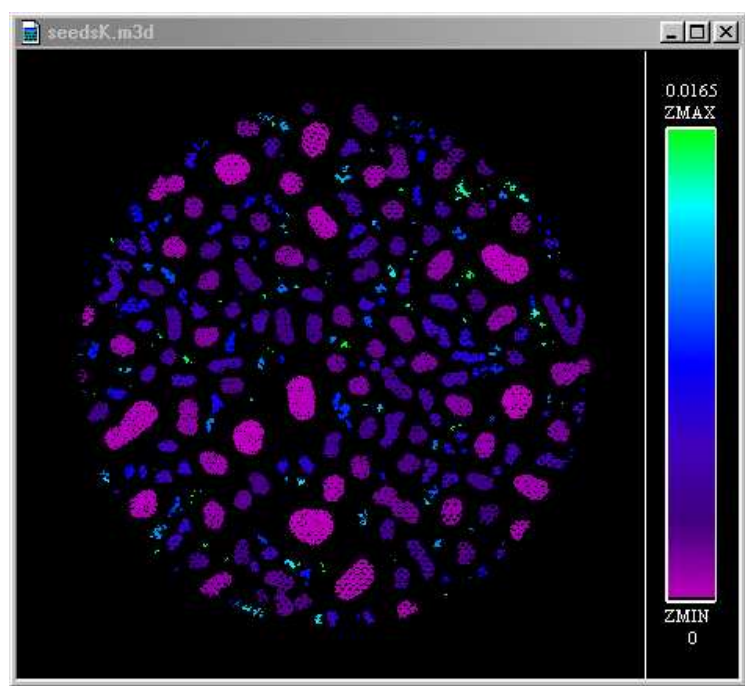

Fig. 14. Calculated seed regions for the 020117b measurement set

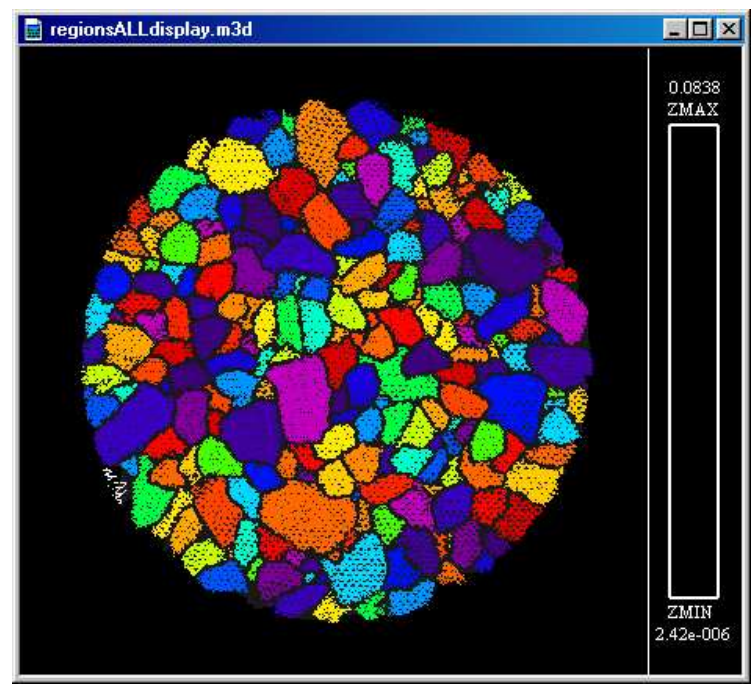

Fig. 13. Accurate segmentation for comparison to figure 15 . Watershed of the manually specified segmentation identifying all of the surface rock fragments in the $020117 \mathrm{~b}$ measurement set

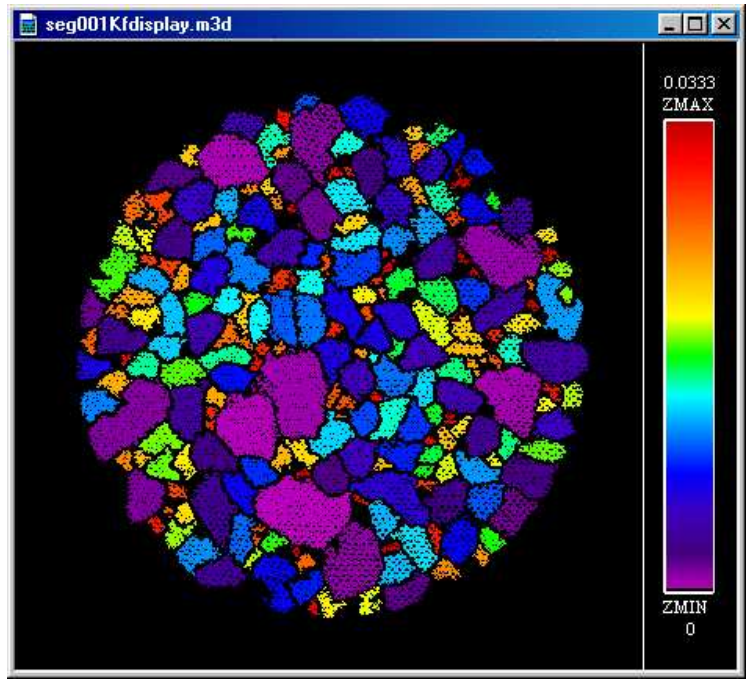

Fig. 15. Calculated segmentation, approximating the visible fragments for the $020117 \mathrm{~b}$ measurement set 


\section{Conclusion}

3D coordinate data of the surface of rock piles has been segmented to identify the surface fragmentation and compared to the individual surface rock fragments in order to determine a measure of error and quantify the results. This comparison has been made possible using visualisation software developed in this research that allows the surface fragments to be mapped into the 3D surface data.

The advantages for fragmentation measurement using image analysis are significant and include; quantifying image segmentation performance in isolation of the downstream processes of fragment classification and size distribution calculation, utilisation of 3D data to overcome various limitations of photographic-based image analysis, and the capacity to use 3D fragment data to eliminate the misclassification of partially visible fragments as smaller entirely visible fragments.

Mathematical definitions for morphological erosion and dilation have been extended from greyscale image based definitions to apply to irregularly spaced 3D surface data. These definitions have been developed into a library of mathematical morphology, image segmentation and supporting algorithms.

3D coordinate surface data can now be morphologically processed directly in 3D , segmented, visualised, and quantified by compared to the actual surface segmentation.

There are several logistical and methodological requirements for collecting 3D data and applying the presented segmentation algorithms that are summarized as follows:

M.1 A 3D range data measurement system for larger, more dynamic and dusty environments is required in order to apply the presented techniques in an industrial application.

M.2 Sufficient data density is also imperative as it defines the smallest fragment size that the measurement system can detect. Data density has been substantially increased in the submitted paper by using data collected from multiple positions. The presented image analysis algorithms have been developed to handle multiple sets of overlapping 3D data.

M.3 For any image analysis technique that one can apply there is a lower limit to the fragment size that can be observed. Given the resolution lower size limit of a particular technique, errors will be introduced if it is used on a rock pile that contains fragments smaller than this limit. The submitted paper does not provide any solutions to dealing with small particles or fines. As mentioned in point M.2, the lower limit of fragment size depends of the density of measured 3D data. Every effort to increase data density 
should be made to increase the range of small fragment sizes that can be measured.

Further advances are necessary to achieve the overall goal of fragmentation measurement.

R.1 Further work to determine the entirely visible and partially visible fragments from the 3D segmentation has been successfully completed by Thurley [28]. Such a capability provides a significant advantage in the classification of fragments over photographic-based methods as it eliminates bias due to the treatment of partially visible fragments as if they were smaller entirely visible fragments.

R.2 Techniques that relate regions in the segmentation into measurements of fragment sieve size are also necessary. A technique based on the best-fit rectangle of Wang et al. [42] has been applied to the presented 3D data by Thurley [28].

R.3 Research to determine what the surface fragmentation of a rock pile indicates about the size distribution of the overall pile is vital. If the surface of the pile is not in some measurable way, representative of the size distribution of the pile, then no matter how accurate the techniques used to analysis the surface, the results can have little relevance to the size distribution of the pile. A mathematical model that relates the proportions visible on the pile surface with the pile size distribution has been developed and applied to laboratory rock piles by Thurley [28] and Thurley and $\mathrm{Ng}[29]$.

\section{References}

[1] J.R. Grant, T.N. Little, and D. Bettess. Blast driven mine optimisation. In Explo 1995 Explo 1995 [43], pages 3-10.

[2] T. Kojovic, S. Michaux, and C. McKenzie. Impact of blast fragmentation on crushing and screening operations in quarrying. In Explo 1995 Explo 1995 [43], pages 427-436.

[3] N.H. Maerz, J.A. Franklin, L. Rothenburg, and D. Coursen. Measurement of rock fragmentation by digital photoanalysis. In Proceedings of the Congress of the International Society of Rock Mechanics, volume 6(1), pages 687-692, 1987.

[4] W.X. Wang and O. Stephansson. Comparison between sieving and image analysis of aggregates. In Franklin and Katsabanis [44], pages 141-148.

[5] K.M. Grainger and G.G. Paine. The development and application of a photographic fragmentation sizing assessment technique for blast analysis. In FRAGBLAST 90 Proceedings, pages 255-258, Brisbane, Queensland, Australia, August 1990. 
[6] J.A. Franklin, T. Katsabanis, and A. Rustan. Preface. In Franklin and Katsabanis [44], pages 3-4.

[7] S. Dahlhielm. Industrial applications of image analysis - The IPACS system. In Franklin and Katsabanis [44], pages 59-65.

[8] D.C. Downs and B.E. Kettunen. On-line fragmentation measurement using the CIAS system. In Franklin and Katsabanis [44], pages 79-82.

[9] T. Havermann and W. Vogt. TUCIPS - A system for the estimation of fragmentation after production blasts. In Franklin and Katsabanis [44], pages $67-71$.

[10] T.H. Kleine and A.R. Cameron. Blast fragmentation measurement using Goldsize. In Franklin and Katsabanis [44], pages 83-89.

[11] N.H. Maerz. Reconstructing 3-d block size distributions from 2-d measurements. In Franklin and Katsabanis [44], pages 39-43.

[12] N.H. Maerz, T.C. Palangio, and J.A. Franklin. Wipfrag image based granulometry system. In Franklin and Katsabanis [44], pages 91-99.

[13] J. Schleifer and B. Tessier. Fragscan: A tool to measure fragmentation of blasted rock. In Franklin and Katsabanis [44], pages 73-78.

[14] J. Schleifer and B. Tessier. Fragmentation assessment using the Fragscan system: Quality of a blast. In Explosives in Mining Conference Explo 2001 [45], pages 365-372.

[15] W.X. Wang and O. Stephansson. On-line system for monitoring the size distribution of aggregates on a conveyor belt. In Franklin and Katsabanis [44], pages 167-.

[16] J.M. Kemeny, A. Devgan, R.M. Hagaman, and X. Wu. Analysis of rock fragmentation using digital image processing. Journal of Geotechnical Engineering, 119(7):1144-1160, July 1993.

[17] J.M. Kemeny. Practical technique for determining the size distribution of blasted benches, waste dumps and heap leach sites. Mining Engineering, pages 1281-1284, November 1994.

[18] K.K. Girdner, J.M. Kemeny, A. Srikant, and R. McGill. The split system for analyzing the size distribution of fragmented rock. In Franklin and Katsabanis [44], pages 101-108.

[19] J. Kemeny, E. Mofya, R. Kaunda, and P. Lever. Improvements in blast fragmentation models using digital image processing. In Explosives in Mining Conference Explo 2001 [45], pages 357-363.

[20] N.H. Maerz. Image sampling techniques and requirements for automated image analysis of rock fragmentation. In Franklin and Katsabanis [44], pages $115-120$.

[21] T.C. Palangio and J.A. Franklin. Practical guidelines for lighting and photography. In Franklin and Katsabanis [44], pages 111-114.

[22] N.H. Maerz and W. Zhou. Optical digital fragmentation measuring systems - inherent sources of error. FRAGBLAST - International Journal of Blasting and Fragmentation, 2:415-431, 1998.

[23] N. Cheimanoff, R. Chavez, and J. Schleifer. Fragscan: A scanning tool for fragmentation after blasting. In Proceedings of the Fourth International 
Symposium on Rock Fragmentation by Blasting - FRAGBLAST 4, pages 325-330, Vienna, Austria, July 1993.

[24] I. Frydendal and R. Jones. Segmentation of sugar beets using image and graph processing. In ICPR 98 Proceedings - $14^{\text {th }}$ International Conference on Pattern Recognition, volume II, pages 16-20, Brisbane, Australia, August 1998.

[25] C.V.B. Cunningham. Keynote address: Optical fragmentation assessment - a technical challenge. In Franklin and Katsabanis [44], pages 13-19.

[26] J.M.R Fernlund. The effect of particle form on sieve analysis: a test by image analysis. Engineering Geology, 50:111-124, 1998.

[27] Q. Liu and H. Tran. Comparing systems - Validation of Fragscan, Wipfrag and Split. In Franklin and Katsabanis [44], pages 151-155.

[28] M. J. Thurley. Three Dimensional Data Analysis for the Separation and Sizing of Rock Piles in Mining. PhD thesis, Monash University, December 2002. http://image3d6.eng.monash.edu.au/thesis.html.

[29] M.J. Thurley and K.C. Ng. Modelling the relationship between the surface fragments and the pile size distribution in laboratory rock piles. In submission.

[30] B. F. Alexander and K. C. Ng. 3-D shape measurement by active triangulation using an array of coded light stripes. SPIE Optics, Illumination and Image Sensing for Machine Vision II, 850:199-209, 1987.

[31] G. Matheron. Random Sets and Integral Geometry. Wiley, New York, 1975.

[32] J. Serra. Image Analysis and Mathematical Morphology. Academic Press, 1982.

[33] J. Serra. Introduction to mathematical morphology. Computer Vision, Graphics and Image Processing, 35:283-305, 1986.

[34] S.R. Sternberg. Grayscale morphology. Computer Vision, Graphics and Image Processing, 35:333-355, 1986.

[35] R.M. Haralick and L. Shapiro. Computer and Robot Vision, volume 1, chapter 5, pages 157-260. Addison Wesley, 1992.

[36] S. Beucher and F. Meyer. Mathematical Morphology in Image Processing, chapter 12, pages 433-481. Marcel Dekker Inc., 1992.

[37] J. Serra and L. Vincent. An overview of morphological filtering. Circuits Systems Signal Process, 11(1):47-108, 1992.

[38] I. Pitas and A. Maglara. Range image analysis by using morphological signal decomposition. Pattern Recognition, 24(2):165-181, 1991.

[39] G. Borgefors. Distance transforms in digital images. Computer Vision, Graphics, and Image Processing, 34:344-371, 1986.

[40] M. de Berg. Computational Geometry: Algorithms \&s Applications, chapter 5.2, pages 97-103. Springer, 1997.

[41] L. Vincent. Grayscale area openings and closings, their efficient implementation and applications. In Proc. EURASIP Workshop on Mathematical Morphology and its Applications to Signal Processing, pages 22-27, Barcelona, Spain, May 1993. 
[42] W. Wang, F. Bergholm, and O. Stephansson. Image analysis of fragment size and shape. In Franklin and Katsabanis [44], pages 233-243.

[43] Explo 1995. Explosives in Mining Conference, Brisbane, Australia, September 1995. AusIMM.

[44] J.A. Franklin and T. Katsabanis, editors. Measurement of Blast Fragmentation - Proceedings of the FRAGBLAST 5 Workshop, 1996. Balkema.

[45] Explo 2001. Explosives in Mining Conference, Hunter Valley, NSW, Australia, October 2001. AusIMM. 